\title{
Arbeit und Arbeitsfront: Ideologie und Praxis
}

Für die „Deutsche Arbeitsfront“ (DAF) markierte „Arbeit“ den Kern ihrer Identität und die ideologische wie politisch-praktische Zielrichtung aller ihrer Aktivitäten. Von Anfang an machten Robert Ley, der Leiter der am 10. Mai 1933 gegründeten DAF, und seine Mitstreiter aus dem Programm, dem die neue Organisation folgen sollte, kein Geheimnis. Bereits der Name der neuen Organisation zeigt an, was deren Ziel sein sollte: Nicht eine „Arbeiterfront“, sondern eine „Arbeitsfront“ wollten Ley und seine Entourage aufbauen. Deutlich gemacht wurde damit, dass es dem NS-Regime und seinen Protagonisten nicht um den einzelnen „Arbeiter“ ging und nicht um die Vertretung der inner- und überbetrieblichen Interessen der Arbeitnehmerschaft in einer Gesellschaft, die zwischen 1933 und 1945 weiterhin industriekapitalistisch geprägt blieb. ${ }^{1}$ Für den einzelnen Arbeiter sowie dessen Bedürfnisse und Interessen interessierten sich Ley und andere Protagonisten der Diktatur nicht. Ihnen ging es um „Arbeit“ als ökonomische Substanz, um die Mobilisierung von Arbeit, und zwar nicht um ihrer selbst willen, sondern zielgerichtet. Auch das fand bereits im Organisationsnamen seinen Ausdruck. Denn zweiter Bestandteil des Organisationsnamens war die „Front“. Damit waren Ziel und Mittel markiert, nämlich eine „Militarisierung der Arbeit“ unter dem Primat des Bellizismus, also die Konzentration aller Kräfte auf Aufrüstung, Kriegführung und Kriegswirtschaft.

Als Erfinder des Organisationsnamens gilt Reinhold Muchow, der im August 1933 verstorbene Spiritus Rector der Nationalsozialistischen Betriebszellenorganisation (NSBO). Muchow bezeichnete am 1. März 1933 in einem Leitartikel der Zeitschrift „Arbeitertum“, die bis Mitte 1933 das Zentralorgan der NSBO, danach eines der wichtigen Periodika der DAF war, die „Formierung einer neuen Arbeitsfront“" als Ziel. Das hieß zunächst nichts anderes, als dass eine Organisation aufgebaut werden sollte, die sich „die Vernichtung des Klassenkampfes“ auf die Fahnen geschrieben habe. ${ }^{2}$ Vor dem 2. Mai 1933 sprachen

1 Resümierend: Christof Buchheim, Unternehmen in Deutschland und NS-Regime 1933-1945, in: HZ 282 (2006), S. 351-390; Jochen Streb, Das nationalsozialistische Wirtschaftssystem: indirekter Sozialismus, gelenkte Marktwirtschaft oder vorgezogene Kriegswirtschaft?, in: Werner Plumpe/Joachim Scholtyseck (Hrsg.), Der Staat und die Ordnung der Wirtschaft. Vom Kaiserreich bis zur Berliner Republik, Stuttgart 2012, S. 61-83.

2 Vgl. Daten zur Vorgeschichte der DAF: Wolfgang Pohl, Geschichte der Deutschen Arbeitsfront (5.5.1943), in: Sozialstrategien der Deutschen Arbeitsfront, hrsg. v. der Hamburger Stif- 
die meisten DAF- und NSBO-Funktionäre noch von „Arbeiterfront“. Sie taten dies auch, um Illusionen unter den Arbeitern und Angestellten zu schüren, man wolle anstelle der in Richtungsgewerkschaften zersplitterten Arbeitnehmerverbände der Weimarer Republik endlich die lang ersehnte Einheitsgewerkschaft aufbauen. Das jedoch war nie Absicht gewesen. Am Abend des 2. Mai, nach der insgesamt reibungslos verlaufenen Zerschlagung der freien Gewerkschaften, schien es Ley denn auch nicht mehr nötig, sich zu verleugnen. Robert Ley, als Person Garant eines strikt antigewerkschaftlichen Kurses und deshalb an die Spitze der neuen Organisation berufen, ${ }^{3}$ sprach nun von der ,großen Front der Arbeit“" und von „Arbeitsfront“. ${ }^{4}$

Konzipiert war die am 10. Mai 1933 auf einem bombastischen Kongress förmlich konstituierte neue Organisation ausdrücklich als Gegenprogramm zu den Gewerkschaften. Daraus machten die maßgeblichen Akteure keinen Hehl. Bis zum 2. Mai 1933 hatten Hitler, Ley, Muchow und andere führende Nationalsozialisten den Gewerkschafts-Begriff zumeist mit positiver Konnotation verwendet. ${ }^{5}$ Seit dem 10. Mai erhielten die Begriffe „Gewerkschaften“ und „gewerkschaftlich“ in Reden, Schriften und Periodika der DAF und anderer nationalsozialistischer Institutionen und Organisationen pejorativ konnotierte Bedeutungsinhalte. Die Gewerkschaften und überhaupt „gewerkschaftliches Denken“ galten nun als „Schlacke der Klassengesellschaft“, die es rigoros auszumerzen gelte. ${ }^{6}$

In den folgenden Abschnitten werden zunächst Arbeitsbegriff und ArbeitsIdeologie der Arbeitsfront umrissen. Einzugehen ist in diesem Zusammenhang auch auf das „Gesetz zur Ordnung der nationalen Arbeit“ (AOG) vom 20. Januar

tung für Sozialgeschichte des 20. Jahrhunderts, bearb. v. Michael Hepp et al., Teil B (Microfiche), München 1992, B/2, Nr. 116, Bl. 593-659, hier S. 4. Nach Cornelia Schmitz-Berning (Vokabular des Nationalsozialismus, Berlin/New York 1998, S. 135) findet sich der Terminus „Arbeitsfront“ bereits 1919, in der von Dietrich Eckart - dem Mitbegründer der NSDAP und ersten Herausgeber des Völkischen Beobachters - herausgegebenen Zeitschrift „Auf gut Deutsch“.

3 Bereits als NSDAP-Gauleiter für Köln-Aachen (von Juli 1925 bis Mai 1931) hatte sich Ley scharf gegen Gewerkschaften positioniert. Am 9.12.1932 war es nicht zufällig der strikt antigewerkschaftliche Ley, der Gregor Strasser als Stabsleiter (später: Reichsorganisationsleiter) der NSDAP ablöste, nachdem dieser kurz zuvor Gewerkschaften als unabdingbar für den „neuen Staat" bezeichnet hatte.

4 Rede vom 2.5.1933, in: Sebastian Heuel, Der umworbene Stand. Die ideologische Integration der Arbeiter in den Nationalsozialismus 1933-1935, Frankfurt a.M./New York 1988, S. 623-628, hier S. 628.

5 Dies macht z. B. die Lektüre der Passagen von Hitlers „Mein Kampf“ deutlich, in denen dieser sich über Gewerkschaften auslässt. Vgl. auch den Beitrag von Michael Wildt in diesem Band.

6 Zeitgenössische Zitate. Heuel, Stand, insb. S. 300f. 
1934 und die aktive Beteiligung der DAF-Führung an der Ausformulierung dieses Grundgesetzes der nationalsozialistischen Arbeitsverfassung. Im anschließenden Teil sind die Organisationsprinzipien dieses eigentümlichen Massenverbandes anzusprechen, danach wichtige Elemente der Praxis der Arbeitsfront zu skizzieren. Im resümierenden Schlussteil wird begründet, warum weit über die betrieblichen Produktionsstrukturen hinaus Ökonomie und Gesellschaft während der Hitler-Diktatur Züge eines spezifisch nationalsozialistischen Kriegsfordismus annahmen - und die DAF sich als herausragender Propagandist dieses Kriegsfordismus profilierte.

\section{Das Ziel: für den „Führer“ ein Massenheer an leistungsbewussten „Soldaten der Arbeit“}

Die „Frontgemeinschaft“ der „Schützengräben“ war das Ideal, das Ley immer wieder beschwor, mit nostalgischen Erinnerungen an den Ersten Weltkrieg verwob und den Mitgliedern der DAF als positives Ziel vorgab - entsprechend der Leitlinie Hitlers: „Wir müssen [das] große Gemeinschaftserlebnis [des Ersten Weltkrieges] in unsere große Lebensgemeinschaft hineintragen [...]. Das ist der letzte Sinn der Arbeitsfront.“7 Die Bezeichnung „Arbeitsfront“ signalisiert ein Verständnis von Arbeit und Arbeitnehmern, das diese zu einer armeeähnlichen Manövriermasse im Interesse der Führer der, wie es sinnfällig seit Oktober 1934 hieß: „Volks- und Leistungsgemeinschaft aller Deutschen“, machen wollte. Der Terminus „Arbeitsfront“ und ebenso die Formel „Soldaten der Arbeit“ - letzteres eine Metapher, die auf Hitler zurückgeht ${ }^{8}$ und von Ley seit Herbst 1933 popularisiert wurde - weckt Assoziationen an industrialisierte Massenkriege und an Generalstäbe, die Fronten mit Millionenmassen an Soldaten je nach den ,Bedürfnissen' des Krieges hin- und herschoben. Tatsächlich wollte die nationalsozialistische Führung eine Arbeitnehmerschaft, die sich je nach den ,Notwendigkeiten“ von Aufrüstung und Kriegswirtschaft beliebig manövrieren ließ.

Die Armee der „Soldaten der Arbeit“ wiederum war nicht etwa egalitär konzipiert. Sie sollte wie jede Armee vielmehr intern scharf hierarchisiert sein, auch und gerade unterhalb des „Generalstabes“. Die Unternehmer als „Betriebsfüh-

7 NS 5 VI, Nr. 5872, Rede Hitlers auf dem NSDAP-Reichsparteitag in Nürnberg vom 11.9.1939, nach: Grundstein Nr. 10 vom 10. Okt. 1937, in: Bundesarchiv Berlin (künftig: BArch Berlin)

8 Georg Büchmann (Hrsg.), Geflügelte Worte. Zitatenschatz des deutschen Volkes, 29. Aufl., Berlin 1942, S. 644. 
rer“ machte Ley rhetorisch zu den „Offizieren der Wirtschaft“. Die von der DAF ernannten Betriebsobmänner galten ihm als „Feldwebel“. Und auch die breite Masse der „Soldaten der Arbeit“ war keineswegs einheitlich feldgrau, sondern hatte abgestuft auf je unterschiedlichen „Kommandoposten“ zu stehen. ${ }^{9}$ Wer wo stand in der der „Frontgemeinschaft“ nachempfundenen „Betriebsgemeinschaft", entschied sich nach dem sozialdarwinistisch aufgeladenen Leistungsund Konkurrenzprinzip. Ley machte auch daraus keinen Hehl, etwa wenn er einem an höhere DAF-Funktionäre adressierten Vortrag das Motto „Gemeinschaft ist Auslese" voranstellte. ${ }^{10}$

Die Praxis der Arbeitsfront folgte dieser Rhetorik und der von Ley postulierten verstärkten Binnendifferenzierung der „Betriebsgemeinschaften“, die wie die „Volksgemeinschaft“ stets „Leistungsgemeinschaften“ zu sein hatten. So zielte nicht zuletzt das vehement von der DAF propagierte Postulat einer „gerechten Leistungsentlohnung “ auf die verstärkte Aufsplitterung der Belegschaften. Ihren Höhepunkt fand die auf Segmentierung setzende Lohnpolitik im „Lohnkatalog Eisen und Metall“, der unter maßgeblicher Mitarbeit der Arbeitsfront entwickelt, ab 1942 reichsweit eingeführt wurde und die klassische Eingruppierung der Arbeiter in die drei Qualifikationsstufen ,gelernt', ,angelernt' und ,ungelernt' durch acht Grundlohngruppen ersetzte. Auf diese Weise wurden die Einkommensunterschiede viel stärker als zuvor gespreizt ${ }^{11}$ und die Artikulation eigenständiger kollektiver Interessen der Belegschaften weiter erschwert.

Damit nicht genug. Außerdem postulierte die Arbeitsfront, der Wehrpflicht vergleichbar, eine Arbeitspflicht, der kein entsprechendes Recht auf Arbeit gegenüber stand. Ley brachte das im Mai 1934 in die simple Formel, ,dass alle deutschen schaffenden Menschen, so wie sie ihrer Soldatenpflicht genügen, auch ihrer Arbeitspflicht genügen“ müssten. ${ }^{12}$ Ein „Arbeitsrecht“ habe nur derjenige, der „höchste Leistung“ brachte. Wer sich dem nicht unterwarf, würde dies umgehend zu spüren bekommen: „Wer sabotiert, wird zertreten“, so Ley. ${ }^{13}$

9 Zitate: BArch Berlin, R 3901, Nr. 20644, Bl. 70-77 Rs., hier Bl. 71, (Zweite) Rede Leys auf der 3. Reichsarbeits- und -schulungstagung der DAF vom 4.12.1935.

10 Robert Ley, „Gemeinschaft ist Auslese“ (Rede auf der Eröffnungssitzung der Arbeitskammer Berlin-Brandenburg), in: Informationsdienst (InDie) der DAF vom 6. Nov. 1936 (Nr. 260, Bl. a).

11 Zum Ideologem der „gerechten Leistungsentlohnung“ wie zum „Lohnkatalog Eisen und Metall“: Tilla Siegel, Leistung und Lohn in der nationalsozialistischen „Ordnung der Arbeit“, Opladen 1986, S. 165-269; Marie Luise Recker, Nationalsozialistische Sozialpolitik im Zweiten Weltkrieg, München 1985, S. 223-250 ; Rüdiger Hachtmann, Industriearbeit im Dritten Reich. Untersuchungen zu den Lohn- und Arbeitsbedingungen 1933-1945, Göttingen 1989, S. 161-223. 12 Robert Ley, „Das erste Jahr der Deutschen Arbeitsfront“ (eine Art Rechenschaftsbericht), in: Westdeutscher Beobachter vom 16.5.1934, S. 1-12 (Zitat: S. 9), in: BArch Berlin, NS 22, Nr. 755. 13 Der Abschluß der Reise Dr. Leys, in: Der Deutsche vom 29.4.1934. 


\section{Federführend an der Ausformulierung beteiligt: das „Gesetz zur Ordnung der nationalen Arbeit" und die DAF-Führung}

Ley spitzte zu, was als Grundtendenz in der nationalsozialistischen Arbeitsverfassung angelegt war. Auch deshalb wäre verfehlt, das „Gesetz zur Ordnung der nationalen Arbeit“ (AOG) vom Januar $1934^{14}$ als Niederlage der DAF zu deuten. In der Forschung wird dies gern behauptet, ${ }^{15}$ u. a. mit dem Hinweis darauf, dass die NS-Organisation im AOG keine tarifpolitischen Rechte erhalten habe. Die aber hatten Ley und die Arbeitsfront-Führung nie gewollt. Im Gegenteil, Tarifverhandlungen und damit die Existenz von Tarifkontrahenten galten ihnen als Ausgeburt des „Klassenkampfes“. Bereits auf dem Gründungskongress der DAF vom 10. Mai 1933 hatte Ley die „Erziehung zum Arbeitswillen“ und die „Weckung der Arbeitsdisziplin“ - bei gleichzeitiger „Pflege des Berufsstolzes“ und einer auf das Semantische beschränkten „Veredelung des Begriffes ,Arbeit““sowie die „Erziehung zur Volksgemeinschaft“ als die zentralen Zielen der DAF vorgegeben. ${ }^{16}$ Der Terminus „Arbeit“, so Ley, „ist für uns Nationalsozialisten nicht nur ein wirtschaftlicher Begriff, sondern eine zutiefst weltanschauliche These. Arbeit ist Ausdruck des Lebenskampfes“. ${ }^{17}$ Sich selbst und seine Gefolgsleute erklärte Ley in diesem Sinne zu „Arbeitsfanatikern“, die wollten, dass „der Geist der Volksgemeinschaft die Arbeit durchpulst und durchflutet“.

14 Zum AOG vgl. vor allem: Andreas Kranig, Lockung und Zwang. Zur Arbeitsverfassung im Dritten Reich, Stuttgart 1983; Wolfgang Spohn, Betriebsgemeinschaft und Volksgemeinschaft. Die rechtliche und institutionelle Regelung der Arbeitsbeziehungen im NS-Staat, Berlin 1987; Timothy W. Mason, Zur Entstehung des Gesetzes zur Ordnung der nationalen Arbeit vom 20. Januar 1934. Ein Versuch über das Verhältnis ,archaischer‘ und ,moderner` Elemente in der neuesten deutschen Geschichte, in: Hans Mommsen et al. (Hrsg.), Industrielles System und politische Entwicklung in der Weimarer Republik, Düsseldorf 1974, S. 322-351; Rüdiger Hachtmann, Die rechtliche Regelung der Arbeitsbeziehungen im Dritten Reich, in: Dieter Gosewinkel (Hrsg.), Wirtschaftskontrolle und Recht im Nationalsozialismus - zwischen Entrechtlichung und Modernisierung. Bilanz und Perspektiven der Forschung, Baden-Baden 2004, S. 123-139.

15 So z. B.: Ludolf Herbst, Das nationalsozialistische Deutschland 1933 bis 1945, Frankfurt a.M. 1996, S. 240f.; Hans-Ulrich Thamer, Verführung und Gewalt. Deutschland 1933-1945, Berlin 1986, S. 499; Martin Broszat, Der Staat Hitlers, München 1969, S. 194f.; Ronald Smelser, Hitlers Mann an der „Arbeitsfront“. Robert Ley. Eine Biographie, Paderborn 1989, S.176; Michael Schneider, Unterm Hakenkreuz. Arbeiter und Arbeiterbewegung 1933 bis 1939, Bonn 1999, S. 297. 16 Alle Zitate aus der Rede Leys vom 10.5.1933: Willy Müller, Das soziale Leben im neuen Deutschland, Berlin 1938, S. 67.

17 Robert Ley, Nachlese und Bilanz vom 1.5.1939, in: Der Angriff vom 13.5.1939. 
Das Arbeitsordnungsgesetz wurde von der DAF enthusiastisch begrüßt. So dankte Ley Hitler telegraphisch „von ganzem herzen für das grosszuegige werk, das mit der annahme des gesetzes zur ordnung der nationalen arbeit verwirklicht worden" sei $^{18}$ - nachdem der Wortlaut des AOG definitiv feststand, d.h. eine Woche, bevor das Gesetz am 20. Januar 1934 mit der Veröffentlichung im Reichsgesetzblatt förmlich in Kraft trat. Das war keine vorgetäuschte Begeisterung, die man pflichtschuldig äußerte. Überdies hatte sich die DAF-Führung nicht mit der Rolle des Zaungastes beschieden, sondern das Grundgesetz der NS-Arbeitsverfassung maßgeblich mitgestaltet. Namentlich Rudolf Schmeer und Wolfgang Pohl hatten das Gesetz federführend mitformuliert. ${ }^{19}$ Beide waren Schwergewichte innerhalb der DAF: Schmeer war von Mitte 1933 bis Juni 1936 für die Organisation der NSDAP-Reichsparteitage verantwortlich und neben anderen Funktionen förmlich Stellvertreter Leys als Chef der DAF-Gesamtorganisation sowie Leiter des DAF-Personalamtes. Pohl, der bis 1935 bzw. 1937 hochrangige Posten im Reichsarbeits- und Reichswirtschaftsministerium einnahm, fungierte seit Ende 1933 als persönlicher Referent Leys. Einige Monate nach der Inkraftsetzung des AOG wurde Pohl u.a. für seine Mitarbeit am AOG mit der Leitung des Arbeitswissenschaftlichen Instituts, der Denkfabrik der Arbeitsfront, belohnt. Ley seinerseits erklärte rückblickend, auch er selbst sei „nicht faul“ gewesen und habe bei der Ausformulierung des Arbeitsordnungsgesetzes sogar „gleich die Führung in die Hand“ genommen. ${ }^{20}$

Ausdrücklich begrüßte die DAF-Führung, dass sie nicht in die Rechte der Gewerkschaften eintrat. Die „Treuhänder der Arbeit“, die am 19. Mai 1933 vorläufig und mit dem AOG dann endgültig installiert wurden, erkannte sie als autoritäre tarifpolitische Instanz grundsätzlich an. Allerdings verstand sich die Arbeitsfront von Anbeginn als eine Art politisch-ideologischer Gesamtverantwortlicher und ,Wächter` der „Volksgemeinschaft“, der immer die mit dem da-

18 BA Berlin, R 43 II, Nr. 547, Bl. 102, Telegramm Leys vom 12.1.1934.

19 Zur Beteiligung Schmeers: BArch Berlin, R 3901, Nr. 20644, Bl. 181-193, hier Bl. 182, Schmeers (erste) Rede auf der Arbeitstagung des DAF-Sozialamtes in Würzburg vom 24.-26.7.1937. Pohl verfasste mit einem weiteren „Vater“ dieses Gesetzes auch den maßgeblichen Kommentar: Werner Mansfeld/Wolfgang Pohl (unter Mitarbeit v. Gerhard Steinmann/Arthur B. Krause), Das Gesetz zur Ordnung der nationalen Arbeit. Kommentar, Berlin 1934.

20 Hans-Joachim Reichhardt, Die Deutsche Arbeitsfront. Ein Beitrag zur Geschichte des nationalsozialistischen Deutschlands und zur Struktur des totalitären Herrschaftssystems, Diss. Univ. Berlin 1956, S. 85. Auf den maßgeblichen Einfluss der DAF auf das AOG wies auch die etablierte Presse hin, z. B. die renommierte Deutsche Bergwerks-Zeitung vom 17.1.1934 („Magna Charta der nationalen Arbeit. Soziale Ehre in der Wirtschaftsführung“). 
hinter stehenden gesellschaftlichen Ordnungskonzept verbundenen langfristigen Ziele des Nationalsozialismus im Auge behielt und sich deshalb legitimiert glaubte, auch gegenüber den Treuhändern der Arbeit korrigierend einzugreifen. Aus diesem Selbstverständnis resultierten die von der historischen Forschung ausführlich thematisierten, bis Kriegsbeginn immer wieder aufflackernden Konflikte zwischen den Treuhändern der Arbeit, dem diesen übergeordnete Reichsarbeitsministerium (RAM) und den Organisationen der Wirtschaft $(\mathrm{OgW})$ : Die Arbeitsfront wollte die „Volks- und Leistungsgemeinschaft“ als ,das große Ganze، gewahrt wissen. RAM, OgW und auch die Treuhänder hatten dagegen neben ökonomischen Aspekten (vor allem Senkung der Lohnkosten) in erster Linie den Schutz des einzelnen „Betriebsführers“ im Auge, d.h. die Wahrung der autokratischen Stellung des Unternehmers innerhalb des Betriebes. ${ }^{21}$ Derartigen Partikularinteressen glaubte die DAF-Führung entschieden entgegenzutreten zu müssen. So anerkannte sie grundsätzlich zwar die unbeschränkte Stellung des „Betriebsführers“ in seinem Unternehmen, da anders der verhasste „Klassenkampf“ nicht aus der Welt zu schaffen sei. „Der Unternehmer soll wieder Führer des Betriebs sein, aber wehe ihm, wenn er seinen Führerpflichten nicht genügt.“22 Hinter dieser Drohung Leys stand der Wille, gegen „eigensüchtige“, vermeintlich unsoziale Unternehmer aufzutreten. Dazu gehörten auch solche, die ihre Belegschaften ,unnötig‘ schlecht behandelten, damit Unzufriedenheit und Widerstand provozierten - und so das Ansehen des NS-Regimes zu ruinieren drohten.

Indem die Organisationen der gewerblichen Wirtschaft ihrerseits der Arbeitsfront eine gewerkschaftliche Attitüde unterstellten, kaschierten sie diesen Zusammenhang - und ebenso, dass sie selbst zunächst dem „Klassenkampf“Denken der Weimarer Republik verhaftet blieben. Ihre verbalen Ausfälle gegen die Arbeitsfront lassen leicht übersehen, dass Ley sich von Anfang an gegen NSBO- und DAF-Funktionäre positionierte, die dessen populistische Statements als Ermunterung missverstanden, sich aggressiv-aktionistisch als braune Gewerkschaftler gegen tatsächlich oder vermeintlich unsoziale „Betriebsführer“ aufzuspielen. Ley hatte es nicht leicht, sich in den eigenen Reihen auch tatsäch-

$21 \mathrm{Zu}$ den Treuhändern der Arbeit und ihrer Arbeitgebernähe: die in Anm.14 genannten Studien sowie Rüdiger Hachtmann, Atomisierung, Entmündigung, Zwangsorganisation Arbeitsrecht und Arbeitsverfassung im Dritten Reich, in: Marc von Miquel (Hrsg.), Weichenstellungen im Arbeits- und Sozialrecht. Diktatorische Vergangenheit und demokratische Prägung, Geldern 2013, S. 64-94, insb. S. 65-69. Kurzbiographien aller Treuhänder der Arbeit: ebd., S. 86-94.

22 Ley an die Leitung des 1926 gegründeten DINTA anlässlich dessen freiwilliger Eingliederung in die DAF, vom 26.7.1933, in: Arbeitsschulung [Zentralorgan des DINTA] 4 (1933), S. 73. 
lich durchzusetzen. Noch Ende 1933 klagte er darüber, wie schwierig es sei, den unteren Chargen innerhalb der DAF und der NSBO das „gewerkschaftliche Denken“ auszutreiben und diesen den ganz „neuen Geist der Arbeitsfront“ einzutrichtern. ${ }^{23}$ Bis Herbst 1934 wurden mehrere zehntausend Funktionäre aus der DAF und NSBO hinausgesäubert ${ }^{24}$ und so die Vorgabe Leys, aus der Arbeitsfront jeglichen ,gewerkschaftlichen Geist“ zu tilgen, umgesetzt.

Mit dem AOG (so lässt sich resümieren) wurde festgeschrieben, was die engere DAF-Führung seit Mitte 1933 als Richtschnur vorgegeben hatte. Nur in einer Hinsicht waren Ley und seine Mitstreiter mit dem Arbeitsordnungsgesetz unzufrieden: Sie wollten eigentlich ein kurzes, auf wenige Kernsätze konzentriertes ,Grundgesetz'. Damit jedoch konnten sie sich gegenüber der in traditionellem Rechtsdenken befangenen Ministerialbürokratie noch nicht durchsetzen. Auch der für Ley und seine Entourage typische militaristische Jargon schimmerte in den einzelnen Artikeln des AOG nur gedämpft durch. In der Anrufung des „Frontgeistes“ (Franz Seldte) waren sich die federführenden Akteure allerdings völlig einig. Ein Denken, Sprechen und Schreiben in militärischen Kategorien war unter den maßgeblichen Protagonisten des Regimes selbstverständlich. ${ }^{25}$ Bei Ley war dies freilich besonders ausgeprägt. So galt ihm und seinen Mitstreitern die eigene Organisation als der „Exerzierplatz, auf dem täglich die Gemeinschaft geübt wird“. ${ }^{26}$ Diese sollte die Mitglieder der „Betriebsgemeinschaften“ „drillen und exerzieren“, bis jene „einen gleichen Schritt haben, einen gemeinsamen Marschschritt“. Ein „gleicher Marschschritt“ könne, so Ley, allerdings nur „aus dem Rhythmus des Blutes kommen“. ${ }^{27}$ Denn „der Deutsche“ sei nicht in erster Linie als Arbeiter, sondern als Krieger besonders tüchtig, nach

23 BArch Berlin, NS 5 I, Nr. 256, Rede Leys auf der Reichskonferenz der NSBO vom 20.11.1933, S. 6.

24 BArch Berlin, NS 26, Nr. 283, Sonderrundschreiben Muchows vom 27.7.1933. Muchow spricht von 100000 ausgeschlossenen NSBO-Mitgliedern. Außerdem: Volker Kratzenberg, Arbeiter auf dem Weg zu Hitler? Die nationalsozialistische Betriebszellen-Opposition. Ihre Entstehung, ihre Programmatik, ihr Scheitern 1927-1934, Frankfurt a.M. 1987, S. 267 (der noch höhere Zahlen nennt); Günter Morsch, Arbeit und Brot. Studien zur Lage, Stimmung, Einstellung und Verhalten der deutschen Arbeiterschaft 1933-1936/37, Frankfurt a.M. 1993, S. 112f.

25 Zur Militarisierung der Sprache ab 1933: Rüdiger Hachtmann, Vom „Geist der Volksgemeinschaft durchpulst“ - Arbeit, Arbeiter und die Sprachpolitik der Nationalsozialisten, in: Zeitgeschichte-Online (2010), [http://www.zeitgeschichte-online.de/zol-sprachpolitik-2010], eingesehen 31.1.2014.

26 Robert Ley, Durchbruch der sozialen Ehre. Reden und Gedanken für das schaffende Deutschland, hrsg. v. Walter Kiehl, Berlin 1937, S. 210.

27 BArch Berlin, R 3901, Nr. 20644, Bl.70-77 Rs., hier Bl.72, (Zweite) Rede Leys auf der 3. Reichsarbeits- und -schulungstagung der DAF vom 4.12.1935. 
dem Motto: „Wecke im Deutschen den Soldat[en], und du wirst immer die höchste Leistung und den größten Erfolg aus ihm herausholen können!“ Die DAF müsse deshalb ,die soldatischen Tugenden, die in unserer Rasse und in unserem Volk vorhanden sind, auch für die Organisation unseres Arbeitslebens“" mobilisieren. ${ }^{28}$

\section{Bellizismus, Antisemitismus, Rassismus, Biologismus und November-Syndrom: Kernelemente des Arbeitsbegriffs der DAF}

Von Anbeginn unter bellizistischen und rassistischen Vorzeichen stand auch das vielfältige sozialintegrative Instrumentarium, welches die DAF entwickelte, also die NS-Gemeinschaft „Kraft durch Freude“ (KdF) als die größte Suborganisation der Arbeitsfront, aber auch z. B. die diversen Volksprodukte des DAF-Konzerns und dessen sonstige Dienstleistungen, die die Arbeitsfront zu einem „volksgemeinschaftlichen Dienstleister“ machten. ${ }^{29}$ Symptomatisch ist hier ein vielzitierter Aufruf Hitlers, mit dem dieser am 14. November 1933 die Gründung von KdF zwei Wochen später ankündigte: „Ich will, daß dem Arbeiter ein ausreichender Urlaub gewährt wird [...]. Ich wünsche das, weil ich ein nervenstarkes Volk will, denn nur allein mit einem Volk, das seine Nerven behält, kann man wahrhaft große Politik machen“. Drei Tage später, am 17. November, ergänzte Ley diese Worte seines „Führers“ in einem Artikel der DAF-Zeitschrift „Arbeitertum“, in dem er die Gründung des Freizeitwerkes „Nach der Arbeit“ (aus dem dann Anfang 1934 „Kraft durch Freude“ wurde) mit folgenden Worten ankündigte: „Wir verloren den [Ersten Welt-]Krieg, weil wir die Nerven verloren haben. Der beste Staatsmann kann mit einem Volk mit zerrütteten Nerven keine Politik treiben. Der verlorene Krieg sollte uns diese Erkenntnis für alle Ewigkeit eingehämmert haben. [...] Deshalb will der Führer, daß der nationalsozialistische Staat [...] dafür sorgt, daß die Nerven des Volkes gesund und stark bleiben“. ${ }^{30}$ „Wir haben den [Ersten Welt-]Krieg deshalb verloren [so Ley], weil die

28 Robert Ley, Betrachtungen zum 1. Mai. Der Frontarbeiter, in: Angriff vom 1./2.5.1941.

29 Zum Terminus „volksgemeinschaftlicher Dienstleister“ und zu dem dahinter stehenden Konzept: Rüdiger Hachtmann, Das Wirtschaftsimperium der Deutschen Arbeitsfront, Göttingen 2012, S. 14ff. u. 566f.

30 Ley auf der Sitzung des Kleinen Arbeitskonvents der DAF vom 17.11.1933, in: Informationsdienst der DAF, Ausgabe A, vom 18.11.1933 (Nr. 16, Bl. 1). Zum KdF-Massentourismus vgl. vor 
Gegner bei der Überzahl ihrer Menschen dauernd ihre Soldaten sich erholen und ausruhen lassen und immer wieder frische Menschen in den Kampf schicken konnten, während wir unsere Menschen wochen- und zum Teil monatelang in den Gräben lassen mussten. Diese Menschen verloren ihre Nerven, und in der Heimat war es dasselbe.“ Deshalb habe ihm „der Führer selbst“ die Gründung von $\mathrm{KdF}$,als direkte Aufgabe“ übertragen. ${ }^{31}$

Neben Bellizismus und Militarismus waren Antisemitismus und überhaupt Rassismus zentrale Elemente, die auch die Praxis der DAF nachhaltig strukturierten. Der Antisemitismus der Arbeitsfront besaß seine Wurzel zum einen in der Unterscheidung von „schaffendem“ und „raffendem Kapital“ sowie der Gleichsetzung vom „raffendem Kapital“ mit dem Stereotyp vom „jüdischen Wucherer" (ein antisemitisches Stereotyp, das wesentlich auf Henry Ford zurückgeht ${ }^{32}$ ). Fast wichtiger noch ist, dass die Nationalsozialisten ein spezifisches, antisemitisch aufgeladenes Arbeitsethos entwickelten. Hitler postulierte bereits Anfang der 20er Jahre, nur „Ariertum“ prädestiniere für eine „sittliche Auffassung der Arbeit“ und damit für „Gemeinsinn“..$^{33}$ Sein Adlatus Ley teilte diese Auffassung: „Arbeit ist eine Funktion der [arischen] Rasse““. ${ }^{34}$

Die „arische Rasse“ und der Kollektivsingular „die Juden“ werden hier, bei Hitler wie bei Ley, gegeneinander in Stellung gebracht und als sich ausschlieBende Elemente begriffen. Der Rassismus der Arbeitsfront ging jedoch noch weit über den Antisemitismus hinaus. Er schloss im Prinzip alle Nationen und Völker des europäischen Kontinents ein, und zwar nicht erst seit 1939. Es ist kein Zufall, wenn ausgerechnet das Arbeitswissenschaftliche Institut (AWI), der für strategische Konzepte der DAF verantwortliche Think Tank der Organisation, noch vor Beginn des Zweiten Weltkriegs formulierte, dass ,der vorwiegend ostische oder ostbaltische Mensch in der Regel recht gut, der vorwiegend nordi-

allem: Hasso Spode, Arbeiterurlaub im Dritten Reich, in: Carola Sachse et al., Angst, Belohnung, Zucht und Ordnung. Herrschaftsmechanismen im Nationalsozialismus, Opladen 1982, S. 275-328, sowie zur Einordnung von KdF in die Gesamtgeschichte des Tourismus: Rüdiger Hachtmann, Tourismus-Geschichte, Göttingen 2007, insb. S. $122 f f$.

31 Rede Leys auf der NSBO-Reichskonferenz vom 20.11.1933, S. 19, Anm. 23.

32 Zum Antisemitismus Fords: Christiane Eifert, Henry Ford, Antisemit und Autokönig. Fords Autobiographie und ihre Rezeption in Deutschland in den 1920er Jahren, in: ZHF 6 (2009), 2, S. 209-229, online unter: [http://www.zeithistorische-forschungen.de/16126041-Eifert-2-2009], eingesehen 29.1.2014.

33 Morsch, Arbeit, S. 35ff. u. 50; Klaus Holz, Nationaler Antisemitismus. Wissenssoziologie einer Weltanschauung, Hamburg 2001, S. 359-430; Michael Wildt, Geschichte des Nationalsozialismus, Göttingen 2008, S. 10f.

34 (Erste) Rede Leys auf der 3. Reichsarbeits- und -schulungstagung der DAF vom 3.12.1935 (Anm. 27), Bl. 59 Rs.-65 Rs., hier Bl. 62 Rs. 
sche oder fälische Mensch dagegen weniger für Fließ- und im besonderen Bandarbeit geeignet ist“. ${ }^{35}$

Derartige Positionen hatten Vorläufer, nicht zuletzt erneut in Henry Ford. Aufmerksamen Zeitgenossen war dies nicht entgangen. So vermerkte der Vorstandsvorsitzende der Siemens-Schuckert-Werke Carl Köttgen 1928 beifällig: „Will man objektiv beurteilen, wie monotone Arbeit auf den Menschen wirkt, dann muß man zuerst einmal betrachten, wie die einzelnen Manschen ihrem Temperament gemäß geartet“ seien. „Das Arbeitstemperament ist bei den Menschen [...] verschieden je nach Volkscharakter“. Keiner habe das besser erkannt als der US-amerikanische Automobilkönig, so Köttgen: „Es ist bezeichnend, daß in den Fordschen Betrieben an den fließenden Bändern in der Hauptsache Arbeiter stehen, die [...] aus dem östlichen oder südlichen Europa stammen“ “. ${ }^{36}$

Aus der Temperamentfrage, dem xenophoben Ressentiment und einem latenten Rassismus vor 1933 wurde nach 1933 offener Rassismus - in den Arbeitswissenschaften generell ${ }^{37}$ und hier wiederum in besonderem Maße in den Sektoren der DAF, die für eine NS-spezifische Verwissenschaftlichung der Sozialpolitik sowie die praktische Umsetzung der Arbeitswissenschaften in betriebliches Handeln zuständig waren. Den Ton gab erneut das AWI vor. Ende 1939 verlangte der Brain Trust der DAF: Arbeitskräfte für die Fließfertigung seien bevorzugt aus den „neuerdings ins Reich eingegliederten Gebiete[n]“ anzuwerben. ${ }^{38}$ Rassisch angeblich minderwertige Arbeiter an die Fließbänder, ,hochwertige Facharbeit‘ als Privileg einer ,arischen' Arbeiteraristokratie - diese Devise wurde spätestens seit 1941 in zahllosen Unternehmen der verarbeitenden Industrie auch in die Praxis umgesetzt, besonders extensiv in den rüstungsrelevanten Branchen, wie z. B. Lutz Budraß für die Flugzeugindustrie überzeugend gezeigt hat. ${ }^{39}$

Wie nahe sich nationalsozialistischer Biologismus und Rassismus (geschlechtsspezifische und ,rassisch ' bedingte ,Veranlagungen') waren, lässt sich markant an der Begründung des Arbeitswissenschaftlichen Instituts ablesen,

35 AWI der DAF, Die Einsatzfähigkeit von Arbeitskräften für Fließbandarbeiten, in: dass., Jahrbuch 1939, Bd. I, Berlin 1939, S. 441-452 (Zitat: S. 449).

36 Carl Köttgen, Die allgemeinen Grundlagen der Fließarbeit, in: Beihefte zum Zentralblatt für Gewerbehygiene und Unfallverhütung, Beiheft 12: Fließarbeit, Berlin 1928, S. 100ff., zit. nach: Thomas v. Freyberg, Industrielle Rationalisierung in der Weimarer Republik. Untersucht an Beispielen aus dem Maschinenbau und der Elektroindustrie, Frankfurt a.M. 1989, S. 333.

37 Hachtmann, Industriearbeit, S. 83ff.

38 AWI, Arbeitskräfte, S. 449.

39 Lutz Budraß, Der Junkers-Konzern. Das Programm des Heinrich Koppenberg und die Funktion des Fordismus in der Rüstung des „Dritten Reiches“, in: Regine Bittner/Henning Brüning (Hrsg.), Zukunft aus Amerika. Fordismus in der Zwischenkriegszeit: Siedlung, Stadt, Raum, Dessau 1995, S. 149-161, S. 157. 
warum Frauen angeblich „grundsätzlich“ für „die bis ins kleinste unterteilte und hier im besonderen für die Bandarbeit“ prädestiniert seien: „Die schnell erlernbaren Handgriffe, die auch bald bis ins kleinste beherrscht und fast automatisch ausgeführt werden, binden die Frau mit geringer Denkarbeit nur lose an die Arbeit und vor allem nur lose an den Sinn der Arbeit. Sie kann sich während der Arbeit mit ihren privaten und häuslichen Freuden und Sorgen beschäftigen. [...] Das zwangsläufige Arbeitstempo bei der Bandarbeit kommt den Wünschen der industriell tätigen Frau meistens auch entgegen, da ihr dadurch die Verantwortung einer eigenen Initiative in einer Tätigkeit erspart bleibt, deren Sinn ihr fremd ist. " 40 Damit war eine Handlungsmaxime formuliert, die von Mitte der 30er Jahre bis 1941 in rasch wachsendem Umfang in weiten Teilen der verarbeitenden Industrie zur Praxis wurde, ${ }^{41}$ ehe 1941/42 die Millionenheere der „Fremdarbeiter“ beiderlei Geschlechts neben den deutschen Arbeiterinnen zum zweiten Kern des kriegsfordistischen Rationalisierungsproletariats wurden.

\section{Entmündigung und Atomisierung: zur Organisationspraxis}

$\mathrm{Zu}$ Bellizismus, Antisemitismus, Rassismus generell und ebenso Biologismus sowie den damit verbundenen Exklusions- und Segregationsmustern tritt als weiterer Faktor, der Selbstverständnis, Praxis und auch die Organisationsstrukturen der Arbeitsfront prägte, das von Tim Mason so genannte „Novembersyndrom“ hinzu. Der Terminus verweist auf die Angst vor einer sozialistischen Revolution, vor einer von einer radikalisierten linken Arbeiterbewegung getragenen Umwälzung wie ab November 1918, und schloss den tief sitzenden, pathologischen Hass auf Sozialismus und Kommunismus ein, den die Nationalsozialisten mit breiten bürgerlichen und kleinbürgerlichen Schichten teilten. Dieses in subjektiver Perspektive alptraumhafte Bedrohungsgefühl, das bis 1945 nicht wich, prägte das Alltagshandeln wie die organisatorischen Binnenstrukturen der Arbeitsfront. Die DAF verstand sich eine Art Agentur zur inneren Kolonialisierung vor allem der deutschen Arbeiterschaft, weniger der Angestellten; letztere hatten zu erheblichen Teilen aufgrund einer Sozialisation durch den antisemi-

40 AWI, Zum Arbeitseinsatz der Frau in Industrie und Handwerk, in: dass., Jb. 1940/41, Bd. I, Berlin 1941, S. 373-418 (Zitat: S. 399). Vgl. auch: dass., Arbeitskräfte für Fließbandarbeiten, S. 450 .

41 Zum „Fraueneinsatz“ in der Industrie: Rüdiger Hachtmann, Industriearbeiterinnen in der deutschen Kriegswirtschaft 1936-1944/45, in: GG 19 (1993), S. 332-366. 
tischen, schließlich semifaschistischen Deutschnationalen HandlungsgehilfenVerband, als der größten Angestelltenorganisation der Weimarer Republik, schon vor 1933 starke NS-Affinitäten ausgebildet. ${ }^{42}$ Der Wille, vor allem der Arbeiterschaft „klassenkämpferische“ Ideen ein für alle Mal auszutreiben, machte die DAF nicht nur zu einer Organisation, die frühzeitig ein vielfältiges Instrumentarium von Lockung und Zwang ausbildete. Das Ziel einer unbedingten inneren Kolonisierung der bis 1933 dem Nationalsozialismus gegenüber distanzierten Sozialschichten erklärte auch, warum die Arbeitsfront keinerlei tarifpolitische Kompetenzen besitzen wollte und warum die DAF-Führung ganz bewusst nicht in die Fußstapfen der Gewerkschaften als Tarifkontrahent der Arbeitgeber trat, dagegen die Wiederherstellung der autokratischen Stellung des „Betriebsführers“ gegenüber den zur „Gefolgschaft“ degradierten Belegschaft als grundlegendes Prinzip der NS-Arbeitsverfassung ausdrücklich billigte. Die Arbeiter und Angestellten ,positiv“ zur „Volksgemeinschaft“ zu erziehen, implizierte die Aufhebung jeglicher individuellen und kollektiven Autonomie. Selbst Ansätze dazu mussten unterbunden werden. Die Organisationsstrukturen wie die Politik der DAF zielten auf eine vollständige Entmündigung, und zwar nicht erst der „Fremdarbeiter“, deren außerbetriebliche „Betreuung“ der DAF im Mai 1942 durch den „Generalbeauftragten für den Arbeitseinsatz“ Fritz Sauckel übertragen wurde, sondern bereits der deutschen Arbeiter und Angestellten.

Es war angesichts dieses Ziels der Entmündigung der deutschen Arbeitnehmerschaft nur folgerichtig, dass die Anfang 1934 geschaffenen Reichsbetriebsgemeinschaften (RBG) mit den bis 1933 politisch und auch finanziell starken Einzelgewerkschaften namentlich des ADGB nichts mehr gemein hatten. Die RBG fungierten als wirtschaftsfriedliche Scharniere zwischen den Gliederungen der Selbstverwaltung der Wirtschaft (also der Reichswirtschaftskammer, der Reichsgruppe Industrie, den Wirtschafts-, Fachgruppen usw.) und der DAF. Konsequenterweise wurden die RBG 1937 in „Fachämter“ umbenannt. Es war vor diesem Hintergrund nur folgerichtig, dass die Masse der DAF-Mitglieder selbst nicht in den RBG, sondern unmittelbar zentral in der Arbeitsfront organisiert wurde. Irgendwelche Möglichkeiten, sich selbständig entlang von berufli-

42 Iris Hamel, Völkischer Verband und nationale Gewerkschaft. Der Deutschnationale Handlungsgehilfenverband 1893-1933, Frankfurt a.M. 1967, insb. S. 238-263; Michael Prinz, Vom neuen Mittelstand zum Volksgenossen. Die Entwicklung des sozialen Status der Angestellten von der Weimarer Republik bis zum Ende der NS-Zeit, München 1986, insb. S. 69ff.; zur starken Verankerung dieser NS-Organisation unter Angestellten: Kratzenberg, NSBO, insb. S. 219ff. sowie exemplarisch: Rüdiger Hachtmann/Christoph Kreutzmüller, Arbeiter und Arbeiterorganisationen in Berlin (1930-1945), in: Michael Wildt/Christoph Kreutzmüller (Hrsg.), Berlin 19331945. Stadt und Gesellschaft im Nationalsozialismus, München 2013, S. 111-126, hier S. 113f. 
chen oder branchenbezogenen Gemeinsamkeiten zu organisieren, waren ihnen damit genommen. Franz Leopold Neumann sprach bereits 1942 treffend davon, dass die Arbeitsfront zum „charakteristischen Ausdruck des Prozesses vollkommener Atomisierung der deutschen Arbeiterklasse“ geworden sei. ${ }^{43}$

Die kurzfristig angestrebte passive, „negative Integration“ der Arbeitnehmer sollte sukzessive zu einer „positiven Integration“ erweitert, nämlich durch eine möglichst weitgehende aktive Affirmation des nationalsozialistischen Normensystems und dessen Verinnerlichung als handlungsleitende Maximen abgelöst werden. Ziel war, die Arbeiter und Angestellten deutscher Staatsangehörigkeit und ,arischer Rasse“ dazu zu bringen, aus freien Stücken „dem Führer entgegen zu arbeiten" (Ian Kershaw). In diesem Motiv, die deutsche Arbeitnehmerschaft $\mathrm{zu}$ enthusiastischen, sich selbst mobilisierenden Anhängern des Regimes zu machen, hat der für die DAF typische Sozialpaternalismus seine Wurzeln, der organisatorisch seinen Ausdruck in KdF, im Reichsberufswettkampf, in den Kampagnen von „Schönheit der Arbeit“, aber auch in den zahlreichen Volksprodukten und weiteren Dienstleistungen des DAF-Wirtschaftsimperiums fand. Alle diese ,Wohltaten' selektierten ihrerseits rassistisch und waren an politische Konformität gebunden. ${ }^{44}$ Die Arbeitsfront war über die unmittelbar repressivkontrollierende Seite hinaus, die die Ley'sche Massenorganisation immer auch besaß und während des Krieges weiter verstärkte, eine ,Dienstleistungsorganisation', die sich vorzüglich in die von antisemitischen und rassistischen Stigmatisierungen durchzogene nationalsozialistische „Volks- und Leistungsgemeinschaft“ einpasste - ein ,volksgemeinschaftlichen Dienstleister eben.

\section{Kriegsfordismus - nicht nur ein Produktionsregime}

Schaut man sich Ideologie und Praxis der Arbeitsfront in vergleichender Perspektive genauer an, gewinnt man den Eindruck, dass diese zu erheblichen Teilen auf Prämissen basierten, die der von den Nationalsozialisten bewunderte

43 Franz L. Neumann, Behemoth. Struktur und Praxis des Nationalsozialismus 1933-1944, Frankfurt a.M. 1977 (zuerst 1942/44), S. 480. Sehr früh bereits ganz ähnlich ein Stimmungsbericht in den SoPaDe-Berichten 1934, S. 120. Außerdem: Detlev Peukert, Die KPD im Widerstand. Verfolgung und Untergrundarbeit an Rhein und Ruhr 1933 bis 1945, Wuppertal 1980, S. 217 sowie vor allem: ders., Volksgenossen und Gemeinschaftsfremde. Anpassung, Ausmerze und Aufbegehren unter dem Nationalsozialismus, Köln 1982, insb. S. 136 f., 140, 284-288 u. $294 f f$. 44 Ausführlich: Hachtmann, Wirtschaftsimperium. 
Henry Ford zehn Jahre vor der NS-Machtergreifung vorformuliert hatte. Man muss sich nur einige zentrale Statements vor Augen halten, die dieser in seiner Autobiographie „My Life and Work“ gemacht hat:

Für Ford war es „völlig ausgeschlossen, den Leuten auch nur vorübergehend ihren Willen zu lassen“, ${ }^{45}$ im Einzelunternehmen wie in der Gesellschaft. Das waren Prämissen, wie sie auch der NS-Arbeitsverfassung sowie der Praxis der DAF zugrunde lagen und bereits in den Basistermini des AOG, „Betriebsführer“ und „Gefolgschaft“, zum Ausdruck kamen. Autonome innerbetriebliche Arbeitnehmervertretungen waren in Fords Augen kontraproduktiv, Gewerkschaften überflüssig. Tatsächlich wurden Gewerkschaften erst nach jahrzehntelangen schweren Kämpfen 1941/42 in seinem Konzern zugelassen.

Die Prämisse Fords, dass die unkalkulierbare Menge politische Führer brauche, nämlich „Menschen, die die formlose Masse [...] zu einem gesunden, wohlgebildeten Ganzen umzuformen vermögen“ und ihrerseits „geistig und moralisch [ge]härtet“" seien, ${ }^{46}$ wurde von Friedrich von Gottl-Ottlilienfeld, der im Mai 1924 das Schlagwort „Fordismus“ erfand und popularisierte, treffend als „Führersozialismus“ gefeiert. Der lakonische Satz Fords: „Kein Ausdruck ist abgedroschener als das Wort ,Demokratie““, ${ }^{47}$ hätte gleichfalls auch von Robert Ley oder einem anderen Protagonisten des NS-Regimes kommen können.

Ford und seine Ideen waren in der zweiten Hälfte der 20er Jahre in Deutschland in aller Munde. Seine Autobiographie war Ende 1923 unter dem Titel „Mein Leben und Werk“ auf Deutsch erschienen und gelangte bereits im ersten Erscheinungsjahr in mehreren hunderttausend Exemplaren auf die Büchertische. ${ }^{48}$ Er faszinierte Industrielle sowie weit darüber hinaus fast alle politischen Lager bis hin zur Sozialdemokratie. Nicht zuletzt in der frühen Hitler-Bewegung stieg Henry Ford in seiner Vierfach-Rolle als herausragender Antisemit, als vorgeblich genialer Tüftler, als Propagandist der industriellen Massenfertigung und eines Kapitalismus ohne Krisen zur vergötterten Ikone auf. ${ }^{49}$

45 Henry Ford, Erfolg im Leben, München 1952, S. 71. Es handelt sich dabei um die neu betitelte Nachkriegsauflage vom „Mein Leben und Werk“. Daraus auch die folgenden Nachweise. Im Vergleich zu den Auflagen 1923 bis 1944 wurden in den Auflagen ab 1945 die (offen) antisemitischen Passagen (S. 292-295 bzw. 281-283) stillschweigend gestrichen. Ansonsten wurde der Wortlaut nicht verändert.

46 Ford, Erfolg im Leben, S. 66f.

47 Ebd., S. 170.

48 Anderswo war die Resonanz dagegen weit geringer: Richard Vahrenkamp, Von Taylor zu Toyota. Rationalisierungsdebatten im 20. Jahrhundert, Köln 2010, S. 103, Anm. 15.

49 Rüdiger Hachtmann, „Die Begründer der amerikanischen Technik sind fast lauter schwäbisch-allemannische Menschen“: Nazi-Deutschland, der Blick auf die USA und die „Amerikanisierung“ der industriellen Produktionsstrukturen im „Dritten Reich“, in: Alf Lüdtke et al. 
Das Selbstverständnis der DAF und Fords Konzept von der Einhegung der breiten Arbeitnehmerschaft ähnelten einander frappierend. Auch wenn Ford selbst sich nach bisherigem Erkenntnisstand nie explizit $\mathrm{zu}$ größten NS-Massenorganisation geäußert hat, kann man die Arbeitsfront bereits aufgrund ihrer entschiedenen Frontstellung gegen „Klassenkampf“ und Gewerkschaften sowie aufgrund ihres Willens, keinerlei autonome Organisierung der Arbeitnehmer zuzulassen, sondern diese repressiv-paternalistisch zu lenken, geradezu als Verkörperung des Traums von Ford von einer idealen Arbeitnehmerorganisation betrachten.

Hinzu treten weitere Aspekte, insbesondere eine bemerkenswerte Vorliebe der DAF und anderer für fordistische Produktionsregime. Ökonomischer Hintergrund dieser Affinität war die Aufrüstung 1933/34 eingeleitete und im Spätsommer 1936 forcierte Aufrüstung. Sie legte die Grundlagen für Massenfertigung in großem Maßstab. Seit Einsetzen der Vollbeschäftigung wurde in allen Zweigen der verarbeitenden Industrie in rasantem Maße und immer umfassender einzelbetrieblich ,fordisiert‘. Im Herbst 1942 resümierte dies der führende Manager eines Flugzeugwerkes mit folgenden Worten: „Es ist für uns keine Frage mehr, ob wir irgendwo Fließarbeit anwenden wollen oder nicht. [...] Für uns kann es sich nur um die Frage handeln: Bis wann bringen wir alles zum Fließen und wie bringen wir es noch besser zum Fließen“. 50

In den Jahren zwischen 1935 und 1944, die als erste Blütezeit des betrieblichen Fordismus im deutschen Raum gelten können, ${ }^{51}$ sprach man statt von „Fordismus“ und „Rationalisierung“ zwar lieber von „Leistungssteigerung“ (die auch die schlichte Ausbeutung durch massiven außerökonomischen Druck

(Hrsg.), Amerikanisierung. Traum und Alptraum im Deutschland des 20. Jahrhunderts, Stuttgart 1996, S. 37-66.

50 Nach: Rainer Fröbe, Der Einsatz von KZ-Häftlingen in der Industrie, in: Hamburger Stiftung zur Förderung von Wissenschaft und Kultur (Hrsg.), „Deutsche Wirtschaft“. Zwangsarbeit von KZ-Häftlingen für Industrie und Behörden, Hamburg 1991, S. 33-78, hier S. 45. Zum Verhältnis von Fordismus und Zwangsarbeit: Rüdiger Hachtmann, Fordism and Unfree Labor-Aspects of the Work Deployment of Concentration Camp Prisoners in German Industry between 1941 and 1944, in: IRSH (55) 2010, S. 485-513.

51 Als Überblick sowie zur Einordnung des Dritten Reiches in die Geschichte des Fordismus im 20. Jahrhundert: ders., Fordismus, Version: 1.0, in: Docupedia-Zeitgeschichte, 27. 10. 2011, [http://docupedia.de/zg/Fordismus?oldid=84605], eingesehen 29.1.2014; ders./Adelheid v. Saldern, „Gesellschaft am Fließband“. Fordistische Produktion und Herrschaftspraxis in Deutschland, in: ZHF 6 (2009) 2, S. 186-208. Zum Fordismusbegriff: dies., Das fordistische Jahrhundert. Eine Einleitung, in: ebd., S. 174-185 (auch: [http://www.zeithistorische-forschungen.de/ 16126041-Hachtmann-Saldern-2-2009], eingesehen 29.1.2014, bzw. [http://www.zeithistorischeforschungen.de/16126041-Editorial-2-2009], eingesehen 29.1.2014). 
einschließen konnte) oder von „deutscher Rationalisierung“. Solcherart oberflächliche Umcodierungen konnten das weitere Vordringen tayloristischer und fordistischer Produktionsregime seit Mitte der 30er Jahre freilich nur schlecht kaschieren. Welch hohes Ansehen Henry Ford unter Nationalsozialisten auch nach 1933 genoss, lässt sich u.a. daran ablesen, dass ihm 1938 das Großkreuz des Deutschen Adlerordens verliehen wurde, die höchste Auszeichnung, die die NS-Diktatur an Ausländer zu vergeben hatte. 1940 erschien seine Autobiographie in der 33. Auflage.

Mit der seit Mitte der 30er Jahre staatlicherseits forcierten Normierung und Typenbeschränkung wurden zudem die Voraussetzungen für eine Implementierung fordistischer Produktionsregime weiter verbessert - durch Sonderkommissare, ${ }^{52}$ aber auch unter tatkräftiger Mithilfe der Arbeitsfront. Symptomatisch ist u. a., dass das DAF-,Amt für Betriebsführung und Berufserziehung“ (AfBuB) am 5. Oktober 1935 mit dem „Reichsausschuss für Arbeits(zeit)studien“ (REFA), als der institutionalisierten deutschen Version des Taylorismus, ein Abkommen abschloss, das eine enge Kooperation zwischen beiden Seiten vorsah. Aufgrund dessen gelang es REFA, die Zahl der jährlich ausgebildeten, gerade auch für die Implementierung fordistischer Produktionsregime zentralen Zeitnehmer bis 1942 gegenüber 1929 (dem besten Jahr von REFA während der Weimarer Republik) mehr als zu verzehnfachen. ${ }^{53}$ Das AfBuB war 1935 aus dem Mitte der 20er Jahre gegründeten „Deutschen Institut für technische Arbeitsschulung“ (DINTA) hervorgegangen. Das DINTA wiederum, das von der NS-Machtergreifung enthusiasmiert worden war und sich im Juli 1933 der Arbeitsfront aus freien Stücken angeschlossen hatte, hatte sich bis 1933 auf die Ausbildung von Facharbeitern fokussiert und gleichzeitig, wie REFA, ebenfalls Arbeits- und Ermüdungsstudien sowie Experimente auf dem Feld der Industriellen Psychotechnik usw. durchzuführen begonnen. Darüber hinaus besaß das DINTA, wie REFA, enge Kontakte zum Kaiser-Wilhelm-Institut für Arbeitsphysiologie (KWI-A) in Dortmund. Seine zunächst in kleinem Maßstab durchgeführten Experimentreihen zur „nervösen Überbeanspruchung“ bei dauerhafter Fließbandarbeit, zu „Antriebsmomenten bei weitgehend automatisierten Arbeitsformen“ und anderen Folgewirkungen fordistischer Produktionsregime ${ }^{54}$ weitete das KWI-A, das

52 Als Überblick: Hachtmann, Industriearbeit, insb. S. 72ff. Zur Arbeitsfront als Propagandist fordistischer Produktionsregime vgl. die im Entstehen begriffene DAF-Gesamtdarstellung des Verfassers.

53 Ders., Industriearbeit, S. 175-181.

54 Zum Verhältnis zwischen KWI-A und DINTA bzw. AfBuB sowie der DAF-Gesamtorganisation: ders., David gegen Goliath? Das Kaiser-Wilhelm-Institut für Arbeitsphysiologie und die Deutsche Arbeitsfront, in: Theo Plesser/Hans-Ulrich Thamer (Hrsg.), Arbeit, Leistung 
Zentrum der arbeitswissenschaftlichen Grundlagenforschung in Deutschland, in den 30er Jahre systematisch aus. ${ }^{55}$ Auch das DINTA bzw. AfBuB und die für diese Einrichtung tätigen Arbeitsingenieure fokussierten sich ab 1936 in den verarbeitenden Branchen zunehmend auf das an den Bändern beschäftigte Rationalisierungsproletariat sowie auf die Ausbildung hochwertiger Facharbeiter nun für die neuen Berufsfelder, die die Implementierung fordistischer Produktionsregimes mit sich brachte - als Einrichter, Reparaturhandwerker, Überwacher und Kontrolleure.

Auch z. B. die Aktivitäten und Kampagnen des KdF/DAF-Amtes „Schönheit der Arbeit“ erinnern an Leitlinien Fords, etwa seine Feststellung, dass „die absolute Voraussetzung für hohe Leistungsfähigkeit [...] saubere, helle und gut gelüftete Fabrikräume“ seien. „Ohne Sauberkeit keine Moral“, so Ford. ${ }^{56}$ Ley hätte das nicht besser formulieren können. Leys Pläne für eine „betriebliche Gesundheitsführung“ bis hin zur Kasernierung chronisch kranker, schwer verletzter und invalider Arbeitnehmer hatte Ford gleichfalls vorweggenommen: Verletzte Arbeiter seien „durchaus imstande zu arbeiten“ und müssten nicht krankgeschrieben werden, so Ford. Er war stolz darauf, z. B. „Schwindsüchtige“ in „eigens dazu konstruierten Holzschuppen untergebracht“ zu haben..$^{57}$ Die Reihe der Analogien und konzeptionellen Verwandtschaften zwischen der DAF bzw. dem Nationalsozialismus generell auf der einen und Ford auf der anderen Seite ließe sich fortsetzen.

Der hier eingeführte Terminus „Kriegsfordismus“ unterstellt freilich bereits terminologisch Abweichungen, paradox formuliert, vom „Ford'schen Fordismus“. Entscheidende Differenz war, dass Ford im Rahmen seiner Sozialutopie die moderne Massenkonsumgesellschaft auf Basis einer angeblich krisenfesten Endlosschleife von Massenfertigung und Massenkonsum predigte. Auch den Nationalsozialisten schwebte Ähnliches vor - eine Massenkonsumgesellschaft. Sie wurde von ihnen langfristig durchaus ernsthaft avisiert, sollte freilich rassistisch segregiert sein. Bis 1939 entstanden markante Ansätze einer solchen Mas-

und Ernährung. Vom Kaiser-Wilhelm-Institut für Arbeitsphysiologie zum Max-Planck-Institut für molekulare Physiologie und Leibniz-Institut für Arbeitsforschung in Dortmund, Stuttgart 2012, S. 425-468; ders., Ein Kind der Ruhrindustrie? Die Geschichte des Kaiser-WilhelmInstituts für Arbeitsphysiologie von 1913 bis 1949, in: WestfF 60 (2010), S. 73-154, hier S. 133136. Zum KWI-A während der NS-Zeit: Irene Raehlmann, Arbeitswissenschaft im Nationalsozialismus. Eine wissenschafts-soziologische Analyse, Wiesbaden 2005, S. 98-122 sowie den Beitrag von Irene Raehlmann in diesem Band.

55 Zitat: Hachtmann, Kind, S. 122.

56 Ford, Erfolg im Leben, S. $72 f$.

57 Ebd., S. 70f. 
senkonsumgesellschaft. Der von der NS-Gemeinschaft „Kraft durch Freude“ als der größten Suborganisation der DAF organisierte Massentourismus wies konzeptionell und architektonisch fordistische Züge auf. ${ }^{58}$ Auch die Volksprodukte und sonstigen sozialen Dienstleistungen, die das Wirtschaftsimperium der Arbeitsfront den deutschen „Volksgenossen“ offerierte, verweisen auf das Ziel einer rassistisch segregierten Massenkonsumgesellschaft. Faktisch jedoch blieb die NS-Gesellschaft trotz aller Ansätze ohne elaborierten Massenkonsum und mit ihrem Fokus auf Rüstungsproduktion - kriegsfordistisch. Dies, also ein Primat der Aufrüstung und Kriegswirtschaft bei gleichzeitig erzwungenem Konsumverzicht, mag ab 1939 bzw. 1941 auch für die anderen kriegführenden Industrienationen gegolten haben. Im Unterschied $\mathrm{zu}$ ihnen war der nationalsozialistische Kriegsfordismus jedoch insofern auf höchst fatale Weise einzigartig, als er zudem hochgradig rassistisch aufgeladen war.

Der Fordismus war als Produktionsregime, als gesellschaftsprägendes Konzept wie als Sozialutopie eine markante Signatur der Gesamtgeschichte des „kurzen“ 20. Jahrhunderts. Der Begriff ,nationalsozialistischer Kriegsfordismus“ ist ein wichtiger Aspekt, der den spezifischen Ort der NS-Diktatur innerhalb des „Fordistischen Jahrhunderts“ markiert. Fordismus beschränkt sich dabei nicht auf Fließband, Massenfertigung und Massenkonsum. Antonio Gramsci hat Ende der 20er Jahre hellsichtig beobachtet, dass der Fordismus darüber hinaus u.a. „die Notwendigkeit hervorgebracht [habe], einen neuen Menschentypen auszuarbeiten, der dem neuen Typus der Arbeit und des Produktionsprozesses konform ist““. ${ }^{59}$ Das bezog sich nicht allein auf die ungelernten oder bestenfalls semi-qualifizierten Produktionsarbeiter am Band, sondern mindestens ebenso auf die neuen Facharbeiter mit ihren - im Vergleich zu vorfordistischen, noch stark handwerklich geprägten Tätigkeiten - stark veränderten Berufsfeldern. Ähnlich wie der italienische Faschismus, den Gramsci in erster Linie vor Augen hatte, war auch das Dritte Reich (dies kann hier nur als These formuliert werden) bemüht, einen neuen fordistischen Arbeitertypus auszubilden, der, statt den Werten der alten Arbeiterbewegung von Solidarität und Selbsthilfe zu folgen, einen individualistischen Leistungswillen und ein konkurrenzgeprägtes Karrierebewusstsein an den Tag legte. Statt den Betrieb zum Mittelpunkt seiner Sozialbeziehungen zu machen, rückten für diesen neuen Arbeitertypus außerdem zunehmend Konsum, Tourismus und überhaupt Freizeit ins Zentrum - nach dem

58 Hasso Spode, Fordism, Mass Tourism and the Third Reich. The „Strength through Joy“ Seaside as an Index Fossil, in: JSocH, 38 (2004), S. 127-155; Hachtmann, Tourismus-Geschichte, S. $160 f f$. u. $173 f f$.

59 Antonio Gramsci, Gefängnishefte. Kritische Gesamtausgabe, Bd. IX, Hamburg 1999, hier S. 2069. 
Muster, wie sie KdF organisierte. Insbesondere die Arbeitsfront orientierte in ihren rationalisierenden Bemühungen wie ihren sozialintegrativen Aktivitäten auf „ein beinahe ,amerikanisches` Leitbild des leistungsorientierten Arbeiters“ (Detlev Peukert). ${ }^{60}$ Die Spuren in die Nachkriegszeit sind deutlich sichtbar. ,Amerika‘ wurde nicht erst ab 1945 zum Orientierungsrahmen. Nicht zuletzt die Deutsche Arbeitsfront hat Arbeitsbegriff und ebenso die Mentalitäten zahlloser Arbeitnehmer weit über 1945 hinaus nachhaltig geprägt und dabei unübersehbar Anleihen bei US-amerikanischen Vorbildern gemacht - wenn auch nicht so plakativ wie im Westdeutschland der Nachkriegszeit. Die NS-Organisation hat aus ihrer Orientierung an bestimmten Leitbildern jenseits des Atlantiks jedoch kein schamhaft verhülltes Geheimnis gemacht. Das den Ford'schen Werken von Dearborne nachgebildete und zum DAF-Konzern gehörige Volkswagenwerk am Mittellandkanal ist für die vielfältigen Affinitäten der Arbeitsfront zu Henry Ford und zum Fordismus bis heute das steingewordene Symbol geblieben.

60 Peukert, Volksgenossen, S. 43; ebd., S. 140 u. $287 f$. 